PAEDIATRIC LUNG DISEASE

\title{
Outpatient management of childhood asthma by paediatrician or asthma nurse: randomised controlled study with one year follow up
}

\author{
A W A Kamps, P L P Brand, J L L Kimpen, A R Maillé, A W Overgoor-van de Groes, L C J A M van \\ Helsdingen-Peek, R J Roorda
}

Thorax 2003;58:968-973

See end of article for authors' affiliations

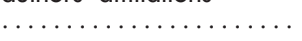

Correspondence to: Dr A Kamps, Department of Paediatrics, Isala Klinieken, Weezenlanden Hospital,', P O Box 10500, 8000 GM Zwolle, The Netherlands; a.kamps@ zonnet.nl

\begin{abstract}
Background: Until now, care provided by asthma nurses has been additional to care provided by paediatricians. A study was undertaken to compare nurse led outpatient management of childhood asthma with follow up by a paediatrician.

Methods: Seventy four children referred because of insufficient control of persistent asthma were randomly allocated to 1 year follow up by a paediatrician or asthma nurse. The main outcome measure was the percentage of symptom-free days. Additional outcome measures were airway hyperresponsiveness, lung function, daily dose of inhaled corticosteroids (ICS), number of exacerbations, number of additional visits to the general practitioner, absence from school, functional health status, and disease specific quality of life.

Results: There were no significant differences at the end of the 1 year study period between the two treatment groups in percentage of symptom-free days (mean difference $2.5 \%$; $95 \% \mathrm{Cl}-8.8$ to 13.8 ), airway hyperresponsiveness $\left(\log _{10} \mathrm{PD}_{20} 0.06 ;-0.19\right.$ to 0.32$)$, functional health status $(10.1 ;-0.3$ to $19.8)$, disease specific quality of life of patients $(0.08 ;-0.9$ to 0.7$)$, and disease specific quality of life of caregivers $(0.09 ;-0.2$ to 0.3$)$, nor in any other outcome parameters. Most outcome parameters improved considerably over the 1 year study period. These improvements were achieved although the daily dose of ICS was reduced by a mean of $26 \%$ compared with the dose received by children at referral. All parents were satisfied with the asthma care received.

Conclusions: After initial assessment in a multidisciplinary clinic, childhood asthma can be successfully managed by an asthma nurse in close cooperation with a paediatrician. During close follow up by paediatrician or asthma nurse, asthma control improved despite a reduction in ICS dose.
\end{abstract}

A ccording to international guidelines, education of patients and their parents is important in the long term management of childhood asthma. ${ }^{12}$ Comprehensive education not only reduces morbidity, emergency visits, and hospital admissions, ${ }^{3-9}$ but also improves quality of life. ${ }^{3}{ }^{10}{ }^{11}$ Asthma nurses are commonly employed to provide this important time consuming task. ${ }^{12-15}$

Follow up in asthma management consists, to a considerable degree, of reinforcement of the patient's and parents' knowledge of the disease, ensuring adherence to the management plan, checking the inhalation technique, and adjustment of the medication according to symptoms of asthma. In principle, all these tasks can be performed by asthma nurses.

Until now, care provided by asthma nurses has been additional to medical care by physicians. Recent evidence suggests that adults with selected chronic diseases can be managed successfully by nurses alone. ${ }^{16-20}$ We designed a randomised trial to establish whether there are differences in efficacy between outpatient management of childhood asthma by paediatricians or by asthma nurses.

\section{METHODS}

Patients

Patients aged 2-16 years newly referred by their general practitioners to the outpatient clinic of the Isala Klinieken (a 1100 bed district general hospital) by general practitioners for chronic persistent asthma were asked to participate in the trial. The patients were categorised as having mild, moderate, or severe asthma based on international guidelines. ${ }^{1}$ We chose to exclude patients under 2 years of age because many patients in this age group wheeze recurrently without having a definite diagnosis of asthma. ${ }^{21}$

All patients were prescribed inhaled fluticasone propionate as maintenance therapy and inhaled salbutamol as needed. The initial dose of fluticasone chosen was dependent on the dose prescribed by the general practitioner and the reported response to it. These drugs were prescribed by dry powder inhaler (Diskus, GlaxoSmithKline) or by metered dose inhaler plus spacer (Volumatic, GlaxoSmithKline), depending on their age. ${ }^{22}$

Patients were not eligible to enter the study if the daily dose of fluticasone propionate required to control their asthma exceeded $500 \mu \mathrm{g}$ (or equivalent doses of beclomethasone or budesonide), if maintenance oral steroids were needed, or if they suffered from a concomitant disease that warranted follow up by a paediatrician. Written informed consent was obtained from patients ( $>12$ years of age) and from all parents. The study was approved by the ethics committee of the Isala Klinieken.

\section{Initial work up}

The initial diagnostic work up for all patients included a full medical history and physical examination by one of the two consultant paediatric pulmonologists (PLPB and RJR). Spirometric tests for children $\geqslant 5$ years of age were performed according to European Respiratory Society guidelines ${ }^{23}$ with a Jaeger Masterlab pneumotachograph (Jaeger Toennies, 
Breda, The Netherlands). Expiratory flow-volume curves were recorded and the forced expiratory volume in 1 second $\left(\mathrm{FEV}_{1}\right)$ was taken from the best of three reproducible manoeuvres and expressed as a percentage of the predicted value $\left(\mathrm{FEV}_{1} \%\right.$ pred $) .^{24}$ The bronchodilator response (BDR) was expressed as the difference between $\mathrm{FEV}_{1} \%$ pred measured before and 20 minutes after inhaling $800 \mu \mathrm{g}$ salbutamol via a metered dose inhaler plus spacer device (Volumatic spacer). Methacholine challenge was performed using a dosimeter method as published previously and expressed as $\mathrm{PD}_{20}$ (dose of methacholine at which $\mathrm{FEV}_{1}$ falls $20 \%$ from baseline)..$^{25}$

In all children allergy to common inhalant allergens was assessed by radioallergosorbent test (RAST) or skin prick test unless this had been performed in the 6 months before inclusion in the study.

\section{Education session}

An asthma nurse conducted a detailed education session with the patient and his or her parents, discussing information about the mechanisms and triggers of the disease, use of controller and reliever medication, management of acute symptoms, when to seek medical advice, and advice on environmental avoidance. Proper inhalation technique was instructed carefully and checked repeatedly until the asthma nurse was satisfied with the patient's inhalation technique.

\section{Randomisation}

Patients were randomly assigned to follow up by either a paediatrician or an asthma nurse. Randomisation was performed using random number tables. Patients were followed up by the same healthcare provider throughout the study.

\section{Follow up}

Patients were seen at regular intervals (1, 3, 6, and 12 months after the start of the study). Additional follow up visits were planned individually if needed according to the judgement of the paediatrician or the asthma nurse. The educational issues described above were addressed at each follow up visit according to a checklist. The duration of the follow up visits was recorded. At the end of the study all patients were seen by the paediatrician.

The nurse led asthma care was provided by two experienced, board certified asthma nurses ( $\mathrm{AO}$ and $\mathrm{LvH})$. The asthma nurse was able to consult the paediatrician at all times to discuss medical issues of patients under her care.

\section{Adjustment of medication}

At each visit, after evaluation of the level of asthma control, predefined adjustments of medication were made according to a protocol. The initial dose of fluticasone was maintained during the first 3 months of the study if adequate control (few symptoms, minimal use of rescue medication $(\leqslant 3$ times per week according to the diary), and no exacerbation of asthma in the last month) of the patient's asthma was achieved. At subsequent visits the dose was tapered off to the lowest effective dose required to maintain control. If the patient's asthma was considered to be inadequately controlled (frequent use of rescue medication, frequent or severe exacerbations of asthma), the dose of fluticasone was doubled. In the remaining cases the dose of fluticasone was continued. No long acting $\beta_{2}$ agonists were applied following Dutch guidelines for maintenance therapy of asthma valid at the time of the study. ${ }^{26}$

If asthma control could not be achieved with fluticasone $500 \mu \mathrm{g} /$ day for patients treated by an asthma nurse, the patient was withdrawn from the study.

\section{Data collection}

Spirometric tests were performed at each regular follow up visit and methacholine challenge at the end of the study period. Patients (or their parents) were asked to record symptoms on a 4 -point scale $(0=$ no symptoms, $1=$ mild symptoms, $2=$ moderate symptoms, $3=$ severe symptoms) and use of rescue medication in a diary for 2 weeks preceding each visit. Information about asthma related absence from school (patients $\geqslant 4$ years) and extra visits to the general practitioner because of respiratory symptoms was also obtained from the patient's diary and double checked at each follow up visit. At the end of the study parents completed a short questionnaire on their satisfaction with the asthma care received.

\section{Outcome measures}

The primary outcome measure was the difference in percentage of symptom free days between the two treatment groups. Secondary outcome measures were lung function, airway hyperresponsiveness, dose of inhaled corticosteroids, use of rescue medication, absence from school, extra visits to the general practitioner, disease specific quality of life, and functional health status.

\section{Functional health status}

The functional health status of the children was assessed at baseline, after 6 months, and at the end of the study. Two validated questionnaires were used-namely, the Dutch parental versions of the Functional Status II (FSII) and the RAND general health rating index. ${ }^{27} 28$ The FSII generic questionnaire consists of 14 questions about the child's behaviour during the previous 2 weeks and the effect of disease on it. The cumulative score lies between 0 and 100, with 100 indicating no behavioural problems. The RAND general health rating index is a 7 item questionnaire. The cumulative score of the items is between 7 and 32. A higher score indicates a better health status.

As a reference group for functional health status, caregivers of healthy classmates of children participating in the study were asked to complete both the FSII and RAND questionnaire.

\section{Disease specific quality of life}

Disease specific QOL was measured, both for patients and their caregivers, at baseline and 6 and 12 months after the start of the study. The Dutch version of the Paediatric Asthma Quality of Life questionnaire (PAQLQ) was used, including 23 items on activity limitation, symptoms, and emotional function. ${ }^{29}$ The Paediatric Asthma Caregiver's Quality of Life questionnaire (PACQLQ) consists of 13 items on the emotional responses of caregivers and interference with family activities. ${ }^{30}$ Answers are expressed on a 7-point scale, a higher score reflecting a better quality of life.

\section{Statistical analyses}

Power calculation was based on the aim to be able to detect a difference of $15 \%$ between groups in the percentage of symptom-free days. Assuming standard deviations of $20 \%$ and a two tailed alpha of 0.05 , a sample size of 70 patients was needed to achieve a power of at least $85 \%$.

Variables were checked for normal distributions. Normally distributed variables are presented as mean (SD) and not normally distributed variables as median (range). $\mathrm{PD}_{20}$ results were logarithmically transformed $\left(\log _{10} \mathrm{PD}_{20}\right)$ to normalise the data and presented as the geometric mean (range). The mean daily dose of fluticasone during the study was calculated for each patient. From the diary, median symptom scores, mean percentage of symptom-free days, and percentage of rescue medication-free days were calculated for each patient and extrapolated over each follow up period. Differences within and between groups were analysed using 
parametric and non-parametric tests depending on the distribution of continuous data, and $\chi^{2}$ tests for proportions. The mean differences between groups in level of $\mathrm{PD}_{20}$, functional health status, and disease specific QOL at the end of the study were adjusted for baseline values (analysis of covariance). SPSS for Windows was used to analyse the data.

\section{RESULTS}

\section{Clinical outcome}

Between December 1999 and December 2000, 74 patients (out of 75 referred) were randomly assigned to follow up by either a paediatrician or asthma nurse. The mother of one patient declined to participate because she was pregnant. Seventy three (99\%) of the patients completed the study. One patient followed up by an asthma nurse was diagnosed with tracheomalacia during the study period and excluded from the study. The baseline demographic and clinical characteristics of patients in both groups were comparable (table 1).

Table 1 Baseline characteristics of patients treated by paediatrician or asthma nurse ${ }^{*}$

\begin{tabular}{|c|c|c|}
\hline & $\begin{array}{l}\text { Paediatrician } \\
(\mathrm{n}=37)\end{array}$ & $\begin{array}{l}\text { Asthma nurse } \\
(n=37)\end{array}$ \\
\hline Age (years) & $6.8(3.5)$ & $5.9(3.6)$ \\
\hline Sex (boys/girls) & $22 / 15$ & $25 / 12$ \\
\hline$\%$ mild persistent asthma & 24.3 & 18.9 \\
\hline$\%$ moderate persistent asthma & 64.9 & 70.3 \\
\hline$\%$ severe persistent asthma & 10.8 & 10.8 \\
\hline Allergy (\% positive) & 64.9 & 56.8 \\
\hline $\begin{array}{l}\text { Family history of asthma (\% } \\
\text { positive) } \dagger\end{array}$ & 48.6 & 40.5 \\
\hline ICS dose ( $\mu \mathrm{g} /$ day) $\S$ & $204(198)$ & $238(233)$ \\
\hline $\mathrm{FEV}_{1}(\%$ pred) $\ddagger$ & $98.7(11.9)$ & $96.6(15.6)$ \\
\hline $\operatorname{BDR}(\%)$ & $7.9(8.1)$ & $12.6(11.3)$ \\
\hline $\log _{10} \mathrm{PD}_{20}(\mu \mathrm{g})$ & $2.1(0.7)$ & $1.8(0.6)$ \\
\hline QOL (caregivers) & $5.9(0.8)$ & $6.1(0.7)$ \\
\hline QOL (children)** & $5.0(1.3)$ & $5.4(1.1)$ \\
\hline FSII score & $65.7(17.2)$ & $76.5(18.9)$ \\
\hline RAND score & $19.6(4.6)$ & $21.3(5.4)$ \\
\hline
\end{tabular}

$\mathrm{BDR}=$ bronchodilator response (change in $\mathrm{FEV}_{1}$ \%pred after bronchodilator); ICS = inhaled corticosteroids; $\mathrm{QOL}$ = quality of life. *Results are presented as mean (SD) unless otherwise specified.

tParent or sibling diagnosed with asthma.

$\ddagger$ Patients $\geqslant 5$ years of age performed lung function measurements $(n=24$ in paediatrician group, $\mathrm{n}=19$ in asthma nurse group).

§Mean daily dose of all patients (fluticasone equivalent)

-Disease specific quality of life of caregivers

**Disease specific quality of life of children $\geqslant 7$ years of age.
The mean percentage of symptom-free days throughout the study was comparable between the two treatment groups (table 2).

Fifty eight $(78 \%)$ of the patients had already been prescribed ICS by their general practitioner before referral (table 1). For these 58 patients the mean dose of ICS at the end of the study was significantly lower than at baseline (215 (168) $v 289$ (207) $\mu \mathrm{g} /$ day; $\mathrm{p}=0.03$, fig 1 ). The maximum dose of fluticasone prescribed during the study was $\leqslant 500 \mu \mathrm{g} /$ day for $99 \%$ of the patients. Only one patient, 16 years of age and followed up by an asthma nurse, was prescribed $1000 \mu \mathrm{g} /$ day after the initial assessment by the paediatrician. At the next visit after I month, asthma control was achieved and the dose of fluticasone tapered off to $500 \mu \mathrm{g} /$ day.

The percentage of patients who demonstrated a correct inhalation technique significantly increased from $65 \%$ to $95 \%$ at the end of the study $(\mathrm{p}<0.0001)$, without a significant difference between the two treatment groups.

$\mathrm{PD}_{20}$ improved significantly during the study and to a comparable level in both groups $(p=0.001$, table 2$)$. There were no significant differences between groups in any of the other clinical end points (table 2). There were no emergency room visits or hospital admissions due to asthma.

\section{Functional health status}

Mean (SD) FSII score of all patients improved significantly from baseline $(75.0(17.4))$ to the end of the study $(87.8$ (14.0); mean difference $12.8 ; 95 \%$ CI 4.5 to $18.5, p=0.002$ ). The mean difference in FSII score between the two treatment groups at the end of the study was 10.1 (95\% CI -0.3 to

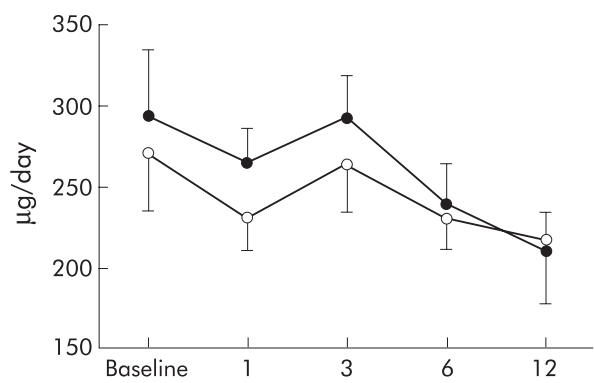

Figure 1 Mean (SE) dose of inhaled corticosteroids (ICS) during the study for patients followed up by paediatrician $(O, n=28)$ and asthma nurse $(\bullet, n=30)$ in patients who had already been prescribed ICS by their general practitioner.

Table 2 Results at end of study for patients treated by paediatrician or asthma nurse*

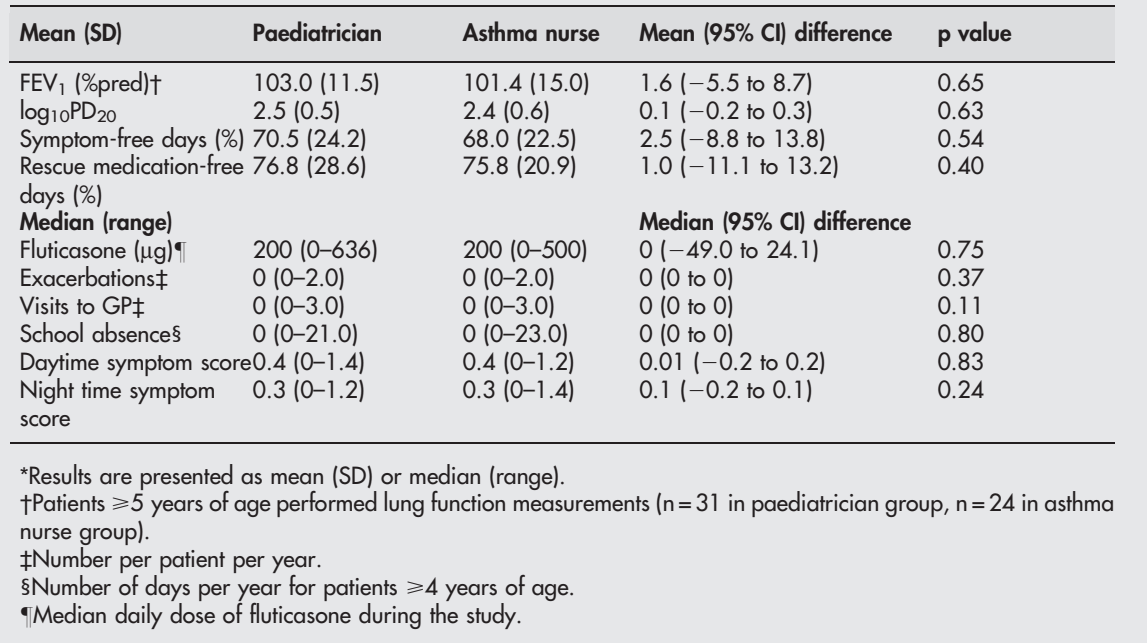




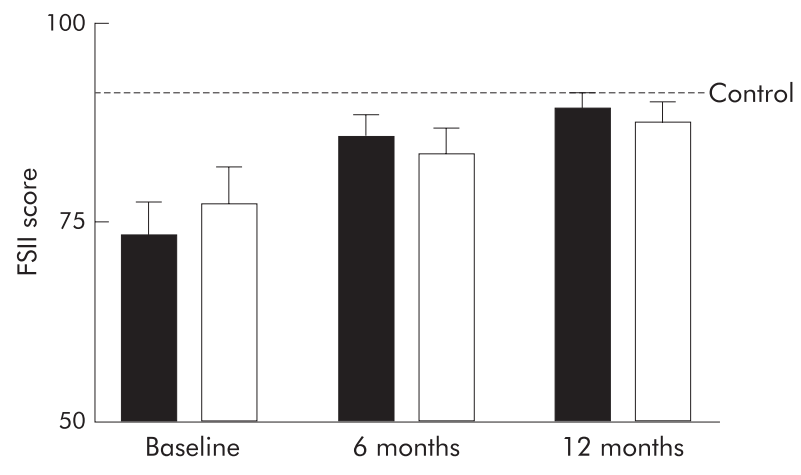

Figure 2 Mean (SE) FSIl score for both treatment groups (closed bars = paediatrician, open bars = asthma nurse) at baseline and after 6 and 12 months of follow up. Dashed line represents the mean FSII score of the control group.

19.8). For the RAND general health rating index the mean (SD) score also significantly improved from 21.3 (4.8) to 24.0 (4.1) (mean difference 2.7; 95\% CI 1.1 to $4.5, \mathrm{p}=0.003$ ) The mean difference in RAND score between both groups at the end of the study was 0.1 (95\% CI -2.8 to 2.7 ).

To put the FSII scores of our study population into perspective, we compared them with the scores of a healthy reference population $(n=57)$. At baseline the mean FSII score of the asthmatic children was significantly lower than that of healthy children $(75.0 \vee 91.0 ; \mathrm{p}<0.001)$, but scores were comparable at the end of the study (87.8 $v 91.0$; $\mathrm{p}=0.06$, fig 2).

At the start of the study patients had significantly lower mean RAND scores than healthy children (21.3 v 27.9; $\mathrm{p}<0.001)$. RAND scores improved significantly in patients, but never reached the level of healthy children (24.0 v 27.9; $\mathrm{p}<0.001)$.

\section{Disease specific quality of life}

The mean overall disease specific QOL score significantly improved throughout the study for both patients (mean difference $0.8 ; 95 \%$ CI 0.4 to $1.3, \mathrm{p}=0.001)$ and their caregivers (mean difference 0.5; 9\% CI 0.4 to 0.7 , $\mathrm{p}<0.0001)$. These improvements in QOL were not significantly different between groups (fig 3). At the end of the study the mean differences in disease specific QOL for patients and caregivers were 0.08 (95\% CI -0.9 to 0.7$)$ and

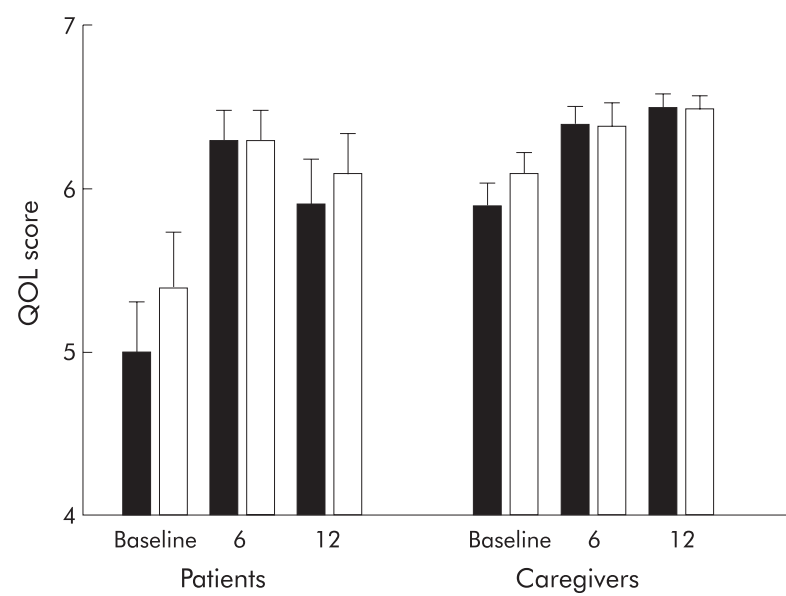

Figure 3 Mean (SE) overall disease specific quality of life score of patients and caregivers at baseline and after 6 and 12 months follow up (closed bars = paediatrician, open bars = asthma nurse).
0.09 ( $95 \%$ CI -0.2 to 0.3 ), respectively. The improvements in the subdomains were also statistically significant and comparable between the two groups (data not shown).

Time investment of paediatrician and asthma nurse

The mean (SD) time spent on patient contact was 136 (14) and 187 (41) minutes for paediatricians and asthma nurses, respectively $(p<0.001)$. During the study period the asthma nurses consulted the paediatrician 22 times for 16 patients representing $43 \%$ of all patients and $8.7 \%$ of all patients' visits to an asthma nurse. This was most commonly for a physical examination in cases of inadequate asthma control.

\section{Parental satisfaction with asthma care}

All parents were satisfied with the asthma care received. In retrospect, $6 \%$ and $3 \%$ of the parents whose child was randomised to follow up by an asthma nurse or paediatrician, respectively, had the desire to have their child seen by a paediatrician more often $(\mathrm{p}=0.51)$. Of the parents whose child was treated by an asthma nurse or paediatrician, $94 \%$ and $82 \%$, respectively, would not object to asthma nurse led management of their child's asthma in the future $(p=0.28)$.

\section{DISCUSSION}

This study shows no differences between outpatient management of children with asthma by a hospital based asthma nurse and traditional management by a paediatrician. No significant differences were found between groups in any of the outcome measures. The study was powered as a comparative trial, not as an equivalence study. The power was sufficient to show that the difference in percentage of symptom-free days between the two treatment groups was unlikely to be larger than $15 \%$. All primary and secondary end points were comparable between groups at the end of the study (table 2); there was no trend in the data to suggest that an increase in power (for example, as an equivalence study) would have yielded different results.

During the study statistically and clinically relevant improvements in airway hyperresponsiveness, functional health status, and disease specific quality of life were seen. In accordance with earlier studies, children with asthma had poorer functional health status at the beginning of the study than their healthy peers, ${ }^{27} 2831$ but by the end of the study they had reached a comparable functional health status. Our study did not have a placebo control group because this was considered to be ethically unjustified. Strictly speaking, therefore, it is possible that the improvements observed in airway hyperresponsiveness, functional health status, and disease specific quality of life are an expression of the natural history of asthma in this cohort, rather than the result of the medical and educational interventions applied. However, the improvements in airway hyperresponsiveness, functional health status, and disease specific quality of life observed in our study are comparable to those found in controlled studies on the effects of education ${ }^{3101132}$ and inhaled fluticasone. ${ }^{33} 34$

Most of the children in our study had already been treated with ICS but had been referred by their general practitioner because of insufficient control of symptoms. After the comprehensive education provided, the percentage of patients with correct inhalation technique increased considerably. This finding confirms our earlier observations that many asthmatic children, even when they have received inhalation instruction in primary care, have poor inhalation technique. $^{35}$ We believe that this improved inhalation technique is an important contributing factor to the improved disease stability experienced by the patients during the study. Importantly, this improvement was achieved without increasing the dose of ICS, which strongly suggests that 
comprehensive patient education and close follow up is very important in the management of childhood asthma.

Many of the children we studied could well be managed in primary care once their asthma is controlled. However, our results emphasise that comprehensive patient education and regular follow up are prerequisites to maintain good asthma control. In our clinic, newly referred patients diagnosed with asthma are asked to visit the outpatient clinic 4-5 times during the first year.

Apart from the expertise of the asthma nurses, we believe that two factors in our study design were important in determining the success of asthma management by asthma nurses. Firstly, the asthma nurse could consult the paediatrician for medical advice, physical examination, or other queries at all times throughout the study. Although this was not a frequent event, the availability of low threshold paediatrician consultation helped to build confidence in the reliability of the nurse led follow up in patients, nurses, and physicians. Secondly, the treatment protocol gave clear guidelines when to increase, reduce, or maintain the dose of ICS, allowing the asthma nurses to adjust treatment independently within certain limits.

Until now, the contribution of the asthma nurse has been complementary to that of the physician. ${ }^{12-15}$ The results of our study clearly show that asthma nurses can take over large parts of long term management of mild to moderate childhood asthma from physicians without compromising quality of care or control of disease. This is in accordance with findings in adults with stable chronic disease in primary and secondary care. ${ }^{16-20}$

Because our study was conducted in a hospital based asthma clinic, our results cannot be extrapolated to a primary care setting. Further studies are needed to evaluate whether asthma management can be performed safely and effectively by asthma nurses alone in primary care. Similarly, the extent to which the results of this study can be extrapolated to other secondary care clinics deserves discussion. In this study, the efficacy of childhood asthma management by a paediatric pulmonologist was compared with care provided by an experienced asthma nurse. In many hospitals routine asthma follow up is provided by general paediatricians or junior doctors. Because it has been shown that specialist care results in better asthma control than management by general paediatricians, ${ }^{36}$ nurse led care may even be superior to doctor led care in such settings. This emphasises the need to train junior doctors adequately in the management of childhood asthma. Finally, because patients with mild intermittent and most severe asthma were not represented in our study population, further studies are needed to assess the efficacy of nurse led asthma care in the extremes of the asthma severity spectrum in children.

In conclusion, we have shown that the efficacy of nurse led outpatient management of childhood asthma was not different from traditional management by a paediatrician. Patients improved considerably despite using a lower dose of ICS, emphasising the importance of comprehensive education and regular follow up. Implementation of such nurse led asthma care will have a considerable impact on the use of healthcare resources.

\section{ACKNOWLEDGMENTS}

This study was sponsored by Isala Klinieken (innovation of care project) and GlaxoSmithKline (unrestricted educational grant for A Kamps).

\section{Authors' affiliations}

A W A Kamps, P L P Brand, A W Overgoor-van de Groes, L C J A M van Helsdingen-Peek, R J Roorda, Department of Paediatrics, Isala Klinieken, Zwolle, The Netherlands
J L L Kimpen, Wilhelmina Children's Hospital, University Medical Centre, Utrecht, The Netherlands

A R Maillé, Julius Center for Health Sciences and Primary Care, University Medical Centre, Utrecht, The Netherlands

Sponsored by GlaxoSmithKline.

A Kamps was supported by an unrestricted educational grant from GlaxoSmithKline. Both P Brand and RJ Roorda have been involved in clinical trials sponsored by GlaxoSmithKline. None of the other authors has any conflict of interest.

A Kamps was the overall study coordinator and was involved in the protocol design, data analysis and interpretation, and in writing the report. P Brand and RJ Roorda were involved in protocol design, data analysis and interpretation, and in writing and editing the report. RJ Roorda and $\mathrm{P}$ Brand were also responsible for clinical care and patient recruitment. A Overgoor and $L$ van Helsdingen-Peek provided clinical care and were involved in editing the report. J Kimpen contributed to the protocol design and was involved in editing the report. A R Maillé was involved in analysis of quality of life data and interpretation, and in editing the manuscript.

\section{REFERENCES}

1 British Thoracic Society, Scottish Intercollegiate Guidelines Network (SIGN). British guideline on the management of asthma. Thorax 2003;58(Suppl I): i1-94

2 National Heart Lung, Blood Institute, National Institutes of Health. International consensus report on diagnosis and treatment of asthma. NHLBI Publication No 92-3091. Eur Respir J 1992:5:601-41.

3 Kelly CS, Morrow AL, Shults J, et al. Outcomes evaluation of a comprehensive intervention program for asthmatic children enrolled in Medicaid. Pediatrics 2000;105:1029-35.

4 Greineder DK, Loane KC, Parks P. Reduction is resource utilization by an asthma outreach program. Arch Pediatr Adolesc Med 1995; 149:415-20.

5 Evans R III, Gergen PJ, Mitchell H, et al. A randomised clinical trial to reduce asthma morbidity among inner-city children: results of the national cooperative inner-city asthma study. J Pediatr 1999;135:332-8.

6 Wesseldine L, McCarthy P, Silverman M. Structured discharge procedure for children admitted to hospital with acute asthma: a randomised controlled trial of nursing practice. Arch Dis Child 1999:80:110-4.

7 Wilson SR, Latini D, Starr NJ, et al. Education of parents of infants and very young children with asthma: a developmental evaluation of the wee wheezers program. J Asthma 1996;33:239-45

8 Ronchetti R, Indinnimeo L, Bonci E, et al. Asthma self-management programme in a population of Italian children: a multicentric study. Eur Respir $J$ 1997; 10:1248-53.

9 Hughes DM, McLeod M, Garner B, et al. Controlled trial of home and ambulatory program for asthmatic children. Pediatrics 1991:87:54-61.

10 Munzenberger PJ, Vinuya RZ. Impact of an asthma program on the quality of life of children in an urban setting. Pharmacotherapy 2002;22:1055-62.

11 Bratton DL, Price M, Gavin L, et al. Impact of a multidisciplinary day program on disease and healthcare costs in children and adolescents with severe asthma. Pediatr Pulmonol 2001:31:177-89.

12 Charlton I, Charlton G, Broomfield J, et al. Audit of the effect of a nurse run asthma clinic on workload and patient morbidity in a general practice. $\mathrm{Br} J \mathrm{Gen}$ Pract 1991;41:227-31.

13 Charlton I, Antoniou AG, Atkinson J, et al. Asthma at the interface: bridging the gap between general practice and a district general hospital. Arch Dis Child 1994;70:313-8.

14 Madge $P$, McColl J, Paton J. Impact of a nurse-led home management training programme in children admitted to hospital with acute asthma: a randomised controlled trial. Thorax 1997;52:223-8.

15 Greineder DK, Loane KC, Parks P. A randomised controlled trial of a pediatric asthma outreach program. J Allergy Clin Immunol 1999;103:436-40.

16 Sharples LD, Edmunds J, Bilton D, et al. A randomised controlled crossover trial of a nurse practitioner versus doctor led outpatient care in a bronchiectasis clinic. Thorax 2002;57:661-6.

17 Mundinger MO, Kane RL, Lenz ER, et al. Primary care outcomes in patients treated by nurse practitioners or physicians. JAMA 2000;283:59-68.

18 Moore S, Corner J, Haviland J, et al. Nurse led follow up and conventional medical follow up in management of patients with lung cancer. BM 2002;325: $1145-7$

19 Lindberg M, Ahlner J, Ekström T, et al. Asthma nurse practice improves outcomes and reduces costs in primary health care. Scand J Caring Sci 2002;16:73-8

20 Hill J, Bird HA, Harmer R, et al. An evaluation of the effectiveness, safety and acceptability of a nurse practitioner in a rheumatology outpatient clinic. Br J Rheumatol 1994;33:283-8.

21 Keeley DJ, Silverman M. Issues at the interface between primary and secondary care in the management of common respiratory disease. 2: Are we too ready to diagnose asthma in children? Thorax 1999:54:625-8.

22 O'Callaghan C, Barry PW. How to choose delivery devices for asthma. Arch Dis Child 2000;82:185-7.

23 Standardized lung function testing: official statement of the European Respiratory Societ. Eur Respir J 1993;16(suppl): 1-100. 
24 Zapletal A. Lung function in children and adolescents. Methods, reference values. In: Zapletal A, Samanak M, Paul T, eds. Progress in respiration research. Basel: Karger, 1987:1 14-218.

25 Birnie DW, thoe Schwartzenberg GWS, Hop WCJ, et al. Does the outcome of the tidal breathing dosimeter methods of assessing bronchial responsiveness in children with asthma depend on age? Thorax 1990;45:199-202.

26 Hoekstra MO. Treatment of asthma in children; revised guidelines by pediatric pneumologists. Section of Pediatric Lung Disease of the Dutch Association of Pediatric Medicine. Ned Tiidschr Geneeskd 1997;141:2223-9.

27 Post MWM, Kuyvenhoven MM, Verheii ThJM, et al. "Functional status II (R)": een vragenlijst voor het meten van de functionele gezondheidstoestand van kinderen. Ned Tijdschr Geneeskd 1998;142:2675-9.

28 Post MWM, Kuyvenhoven MM, Verheij ThJM, et al. "RAND general health rating index for children": een meetinstrument voor de algemene gezondheid van kinderen. Ned Tijdschr Geneeskd 1998;142:2680-3.

29 Juniper EF, Guyatt GH, Feeny DH, et al. Measuring quality of life in children with asthma. Qual Life Res 1996;5:35-46.

30 Juniper EF, Guyatt GH, Feeny DH, et al. Measuring quality of life in the caregivers of children with asthma. Qual Life Res 1996;5:27-34.
31 Stein REK, Jessop D. Functional status II(R). A measure of child health status. Med Care 1990;28:1041-55.

32 Stevens CA, Wesseldine $\sqcup$, Couriel JM, et al. Parental education and guided self-management of asthma and wheezing in the pre-school child: a randomised controlled trial. Thorax 2002:57:39-44.

33 Hofstra WB, Neijens HJ, Duiverman EJ, et al. Dose response over time to inhaled fluticasone propionate treatment of exercise- and methacholineinduced bronchoconstriction in children with asthma. Pediatr Pulmonol 2000;29:415-23

34 Mahajan P, Pearlman D, Okamoto L. The effect of fluticasone propionate on functional status and sleep in children with asthma and on the quality of life of their caregivers. J Allergy Clin Immunol 1998;102:19-23.

35 Kamps AWA, van Ewijk B, Roorda RJ, et al. Poor inhalation technique, even after inhalation instruction, in children with asthma. Pediatr Pulmonol 2000;29:39-42.

36 Diette GB, Skinner EA, Nguyen THT, et al. Comparison of quality of care by specialist and generalist physicians as usual source of asthma care for children. Pediatrics 2001;108:432-7.

\section{LUNG ALERT}

Salt depletion and hypoalbuminaemia in cystic fibrosis may add to hypercapnia in acute respiratory failure

$\Delta$ Holland AE, Wilson JW, Kotsimbos TC, et al. Metabolic alkalosis contributes to acute hypercapnic respiratory failure in adult cystic fibrosis. Chest 2003;124:490-3

$\mathrm{P}$ atients admitted over a 1 year period with acute exacerbations of cystic fibrosis (CF) and hypercapnia $\left(\mathrm{Paco}_{2} \geqslant 45 \mathrm{~mm} \mathrm{Hg}, \mathrm{n}=14\right)$ were compared with a control group with hypercapnic exacerbations of COPD $(n=49)$. The CF group were significantly younger (23 $v 69$ years) with a lower body mass index $\left(18.3 \mathrm{~kg} / \mathrm{m}^{2} v 25.0 \mathrm{~kg} / \mathrm{m}^{2}\right)$ but no significant difference in $\mathrm{FEV}_{1}$ (\% predicted $27 v 34$ ).

Using the Stinebaugh and Austin reference diagram for acid-base classification, a mixed respiratory acidosis and metabolic alkalosis was present in $71 \%$ of patients with $\mathrm{CF}$ and in only $22 \%$ of those with COPD $(\mathrm{p}<0.01)$. Both the plasma chloride and sodium levels were significantly lower in the CF group, together with a greater degree of hypoalbuminaemia.

Metabolic alkalosis is associated with sodium and chloride depletion and is likely to relate to the basic chloride channel defect in CF. A low albumin level may also contribute to a metabolic alkalosis due to its role as a weak non-volatile acid. Resulting respiratory compensation for this metabolic alkalosis results in hypoventilation and increased hypercapnia. This study is the first to show a significant role for a metabolic alkalosis in contributing to hypercapnic respiratory failure in patients with CF. Attention to fluid and electrolyte replacement in this group may aid treatment.

S J Kelly

Specialist Registrar, Adult Cystic Fibrosis Unit, Northern General Hospital, Sheffield, UK kellysj1968@hotmail.com 